\title{
A field-sensitive photoconductive probe for sampling through passivation layers
}

\author{
Jiunn-Ren Hwang, Richard K. Lai, John Nees, Ted Norris, and John F. Whitaker ${ }^{\text {a) }}$ \\ Center for Ultrafast Optical Science, University of Michigan, Ann Arbor, Michigan 48109-2099
}

(Received 20 June 1996; accepted for publication 25 July 1996)

\begin{abstract}
A field-sensitive photoconductive sampling technique has been demonstrated in measurements performed through an insulating layer without the need for conductive contact. Sampled signals are sensed by a virtual-ground, floating-gate amplifier without draining charge from the device under test or the photoconductive switch. The minimum detectable signal is $2.5 \mu \mathrm{V} / \mathrm{Hz}^{1 / 2}$ with a spatial resolution of $7 \mu \mathrm{m}$, while the sampling bandwidth is essentially that observed using photoconductive sampling with a conductive contact to the device under test. The photovoltaic and shot current noise are negligible in comparison with the lock-in amplifier noise since the current flowing in this high-impedance, floating-gate probe is negligible. (C) 1996 American Institute of Physics. [S0003-6951(96)02241-3]
\end{abstract}

Ultrafast sampling technologies based on short-pulse lasers, such as those that utilize the external electro-optic (EO) sampling probe ${ }^{1}$ or the freely positionable, bulk photoconductive (PC) sampling probe, ${ }^{2,3}$ have been developed for internal-node circuit testing. External EO sampling techniques have a sampling bandwidth that can be over $1 \mathrm{THz}$ with a sensitivity limit of several hundred $\mu \mathrm{V} / \mathrm{Hz}^{1 / 2}$ when the fringing field is well coupled into the probe. ${ }^{4,5}$ However, the sensitivity of EO sampling is typically small in comparison with PC sampling, which drains charges directly from a device under test (DUT) when used in its standard, conductive-contact embodiment. Using low-temperaturegrown GaAs (LT-GaAs) substrates, which have a fast carrier trapping time and high mobility, ${ }^{6}$ conductive-contact PCsampling probes can have picosecond temporal resolution and a sensitivity of a few $\mu \mathrm{V} / \mathrm{Hz}^{1 / 2}$. EO sampling has held one advantage over PC sampling for circuit measurements because the former can sense fields that are embedded under insulating passivation layers without requiring conductive contact. In addition, even though a circuit passivation layer might be removed to allow conductive contacts, extracting charge from a DUT in order to gain higher sensitivity might be excessively invasive to devices incorporated in integrated circuits. $^{7,8}$

Recently we have demonstrated a new, epitaxial layer, LT-GaAs sampling probe with a minimum detectable signal of $30 \mathrm{nV} / \mathrm{Hz}^{1 / 2}$. This ultrahigh sensitivity has been realized by reducing the parasitic capacitance and leakage current of the probe. ${ }^{9}$ In this letter, we report that this field-sensitive, PC-sampling probe has the capability to measure picosecond voltage wave forms through passivation layers without charge extraction, yet with a sensitivity of $2.5 \mu \mathrm{V} / \mathrm{Hz}^{1 / 2}$.

Figure 1 shows a schematic diagram of the sampling experiment which was used to measure electrical wave forms through a passivation layer. Two fiber-mounted PC probes were used, one to launch a pulse onto a coplanar stripline (CPS), and one to measure the signal. The CPS had a 20- $\mu \mathrm{m}$ line width and separation on a GaAs substrate, and it served as the DUT. A $0.4-\mu \mathrm{m}$-thick layer of $\mathrm{SiO}_{2}$ was grown over

a)Electronic mail: whitaker@caen.engin.umich.edu half of the CPS to form the passivation layer. At the end of the PC probes, a 7- $\mu \mathrm{m}$-wide Au strip extended beyond the apex of the 1- $\mu \mathrm{m}$-thick LT-GaAs substrate for a distance of $5 \mu \mathrm{m}$. One probe comes in direct contact with the CPS metal line about $40 \mu \mathrm{m}$ away from the passivation layer. The switch on this probe is biased at $5 \mathrm{~V}$, and the probe launches a signal onto the CPS when excited by a short laser pulse. Both conductors of the CPS are connected to the ground to drain away the injected charge. The second fiber-mounted probe, with its electrical output connected to an integrated JFET source follower, is used to sample the launched wave forms. The metal ribbon tip can be positioned along the transmission line, either contacting the conductor of the CPS or the passivation layer over the CPS.

A mode-locked Ti:sapphire laser produces the 100-fs duration pulses used to excite the PC launcher and sampling gate. The launcher beam is split from the original beam and sent through a variable delay and an acoustic-optic modulator driven at frequencies between $50 \mathrm{~Hz}$ and $110 \mathrm{kHz}$. The sampling beam is coupled to the other probe to activate the gate switch. The time response of the launcher switch and the sampling gate are both about $3.5 \mathrm{ps}$ due to the probe

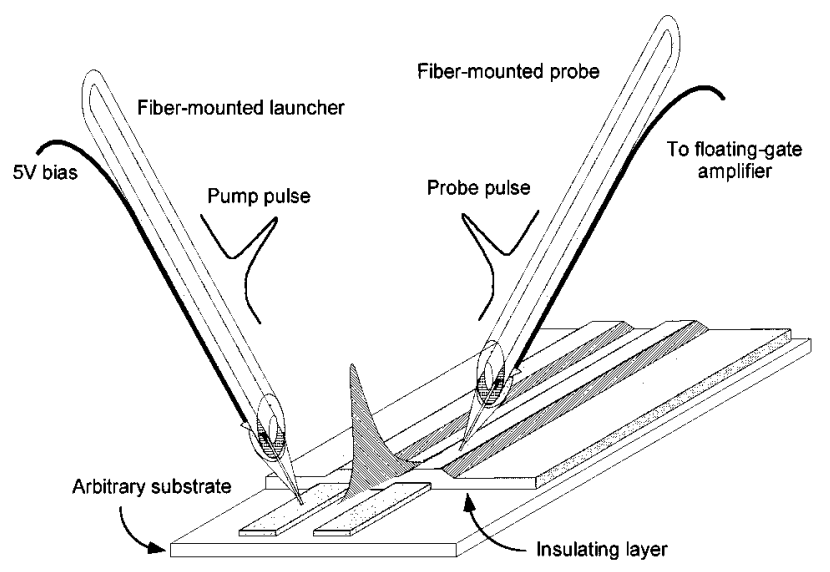

FIG. 1. Schematic diagram of field-sensitive PC sampling through a passivation layer using fiber-mounted, $1-\mu \mathrm{m}$-thick LT-GaAs probes as the wave form launcher and sampler. 


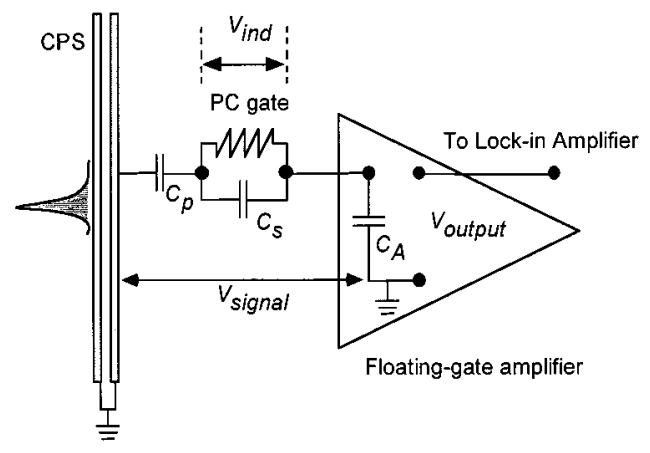

FIG. 2. Lumped-element circuit diagram of the field-sensitive, PC sampling probe with a virtual-ground input, floating-gate amplifier.

design. This is not limited by the response of the CPS, the LT-GaAs, or the single-mode fiber. ${ }^{9}$

The circuit diagram of the field-sensitive PC sampling probe is shown in Fig. 2. Since the dimensions of the metal ribbon tip are much larger than the thickness of the passivation layer, the tip can be considered as a parallel plate capacitor, $C_{p}$. This capacitance is estimated to be about $12 \mathrm{fF}$. The capacitance of the PC gate switch, $C_{s}$, is about $5 \mathrm{fF}$. When the metal ribbon tip has no conductive contact to the DUT, it is essentially a floating metal line with a highimpedance PC switch at its opposite end. Furthermore, the PC gate switch is also floating, but virtually grounded by the extremely high gate input impedance $(<1 \mathrm{~T} \Omega)$ of the integrated source follower/amplifier. A pair of matched, $n$-depletion JFETs are used to provide the source follower. ${ }^{7}$ The capacitance $C_{A}$ is essentially the $3-\mathrm{pF}$ input capacitance of the JFET.

The launched signal traveling along the CPS can be considered as a sheet-charge-density wave passing the floating probe. A sheet-charge density with the same magnitude but opposite polarity is then induced on the probe tip due to their proximity. Similarly, a sheet charge density must be induced at the gate switch $C_{s}$, and again at the capacitance $C_{A}$. Although these elements may not be strictly treated as lumped-element circuit components due to the high sampling bandwidth of the experiment, a lumped-element model does give us insight into this experiment. First, since $C_{A}$ is much larger than $C_{p}$ and $C_{s}$, the sheet-charge-density-induced voltage on $C_{A}$ is negligible, and $C_{A}$ is still at virtual ground. Thus, the induced voltage difference across $C_{s}, V_{\text {ind }}$, is proportional to the signal voltage $\left(V_{\text {signal }}\right)$ and can be given as $V_{\text {ind }}=C_{p} V_{\text {signal }} /\left(C_{p}+C_{s}\right)$. As the PC switch is activated by the gating laser pulse, the photogenerated carriers will sense this differential voltage. A certain amount of charge proportional to the convolution of $V_{\text {ind }}$ and the impulse response of the $\mathrm{PC}$ switch is transferred into $C_{A}$ the same way as in conductive-contact PC sampling. ${ }^{10}$ A static equivalent voltage difference between $C_{p}$ and $C_{A}$ is then established. Here the PC charge injection into $C_{A}$ due to $V_{\text {ind }}$ at each excitation/gate pulse delay time balances the charge returning through the dark current during the time between laser pulses (i.e., $12.2 \mathrm{~ns}$ ). ${ }^{7}$ Since there is no net current through $C_{p}$, the charge deposited into $C_{A}$ will be drained from $C_{p}$. Most of the equivalent voltage will build up at $C_{p}$ due to its

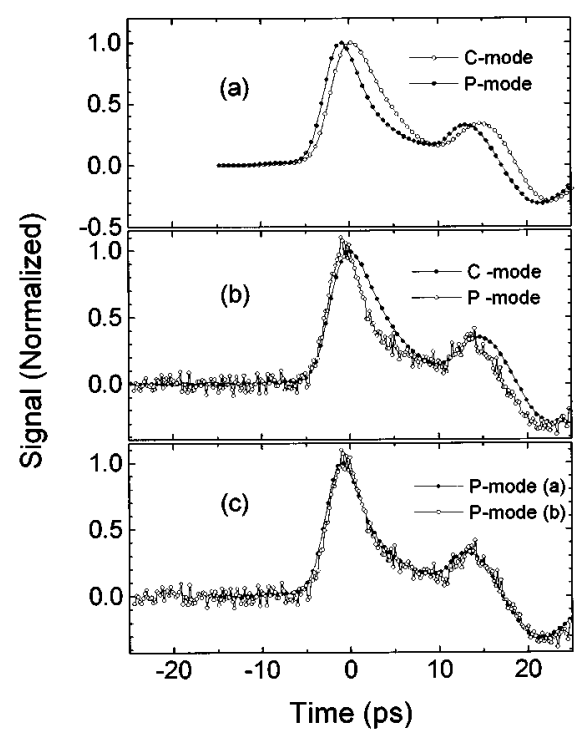

FIG. 3. Normalized time-domain wave forms sampled with the probe in conductive contact (C-mode) or by sensing the field through a passivation layer (P-mode) using $2 \mathrm{~mW}$ of gating laser power. (a) Wave forms for a signal level of several tens of $\mathrm{mV}$; (b) wave forms for a signal level of several tens of $\mu \mathrm{V}$; and (c) direct comparison of wave forms measured through the passivation layer in (a) and (b).

much smaller value than $C_{A}$. The floating voltage of $C_{A}$, which can be read out by the high impedance source follower, is then given as $V_{\text {output }}=C_{p}^{2} V_{\text {signal }} /\left[\left(C_{p}+C_{s}\right)\left(C_{p}\right.\right.$ $\left.\left.+C_{A}\right)\right]$.

Figure 3 shows the normalized picosecond wave forms measured at different signal levels using $2 \mathrm{~mW}$ of gating laser power for both the conductive-contact mode $(C)$ and the field-sensitive mode $(P)$. The propagation distances from the launch site are about $30 \mu \mathrm{m}$ for $C$ mode and $70 \mu \mathrm{m}$ for $P$ mode. The wave forms measured in $C$ mode were obtained using a $1-\mathrm{kHz}$ modulation frequency. These may be considered as reference wave forms, because measurement at this relatively low modulation frequency in conductive-contact mode yields the actual signal amplitude. ${ }^{8}$ The lock-in modulation frequency used for the $P$ mode is much higher, 50 $\mathrm{kHz}$, so that there is lower lock-in-amplifier noise. Figure 3(a) shows a high signal-to-noise measurement for the actual signal wave form, which has a peak amplitude of $19.8 \mathrm{mV}$. The negative peak of the wave forms at a delay of $22 \mathrm{ps}$ is a reflection from wire bonding pads.

By reducing the laser power to the launcher [Fig. 3(b)], we reduced the amplitude of the input signal and were also able to study the sensitivity of the PC probe when measuring through the passivation layer. For an actual signal of $65 \mu \mathrm{V}$ on the CPS, the PC probe senses an amplitude of $180 \mathrm{nV}$ through the passivation layer. The root-mean-square voltage noise of this measurement is about $6 \mathrm{nV} / \mathrm{Hz}^{1 / 2}$, calculated from the base line of the sampled wave form. After normalizing to the actual signal amplitude, the minimum detectable voltage is estimated to be $2.5 \mu \mathrm{V} / \mathrm{Hz}^{1 / 2}$ for the experiment. The high degree of voltage linearity of the field-sensitive PC probe is demonstrated using two normalized time-domain wave forms of different amplitudes [Fig. 3(c)]. Although the signal was reduced by a factor of 400 , the temporal response of the field-sensitive probe does not change. 


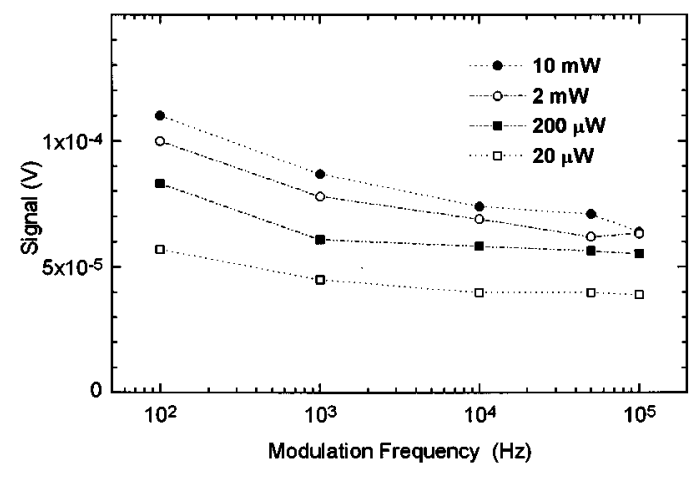

FIG. 4. The peak amplitude of the measured wave forms at lock-in modulation frequencies from $100 \mathrm{~Hz}$ to $110 \mathrm{kHz}$, using gating laser intensities from $10 \mathrm{~mW}$ down to $20 \mu \mathrm{W}$.

One difference in the $C$ mode versus $P$ mode measurement is the response speed. That is, the FWHM of the fieldsensitive wave form is $5.2 \mathrm{ps}$, which is less than the 6.4-ps duration measured in the conductive-contact mode. We believe this is related to the direction of the displacement currents through $C_{s}$. That is, the forward and backward displacement current do not exactly cancel each other due to the presence of the gating pulse. Further study of this interesting effect is underway. However, there is still no net conductive current through $C_{p}$, meaning there is no charge drained from the DUT.

In order to determine the modulation bandwidth of this field-sensitive probe, a wave form with a $30-\mathrm{mV}$ peak amplitude was sampled using different gating laser intensities from $10 \mathrm{~mW}$ down to $20 \mu \mathrm{W}$ and lock-in modulation frequencies from $100 \mathrm{~Hz}$ to $110 \mathrm{kHz}$. Figure 4 shows that the peak magnitude variation of all measurements over this wide range of gating conditions changes by no more than about a factor of 2. This implies that a modulation bandwidth over $110 \mathrm{kHz}$ can be used even for a gating laser power as low as $20 \mu \mathrm{W}$. The high sensitivity at this high modulation bandwidth can be explained by the lumped element model. That is, since most of the static equivalent voltage builds up at $C_{p}$, the effective capacitance $\left(C_{\mathrm{eff}}^{-1}=C_{p}^{-1}+C_{A}^{-1}\right)$ is greatly reduced and is only $12 \mathrm{fF}$ for $C_{p} \ll C_{A}$.

The frequency dependence of the PC gate noise at different gating laser intensities has also been investigated in order to identify the dominant noise source. It was found that the noise level changed from about $12 \mathrm{nV} / \mathrm{Hz}^{1 / 2}$ at $100 \mathrm{~Hz}$ modulation frequency down to $6 \mathrm{nV} / \mathrm{Hz}^{1 / 2}$ at $100 \mathrm{kHz}$ without a noticeable dependence on gating intensity. We conclude that this noise floor arises from both the lock-in amplifier and the integrated JFET source follower. The $1 / f$ photovoltaic noise due to the gating laser amplitude fluctuations for the conductive PC sampling ${ }^{8,11}$ is smaller than the noise of the sampling electronics since the PC-sampling probe is virtually floating and has no closed current path. In general, this field-sensitive probe is free of current noise at the gating laser power level used.

In conclusion, we have demonstrated a fast-response PC sampling method that has a sensitivity of $2.5 \mu \mathrm{V} / \mathrm{Hz}^{1 / 2}$ without draining any net current from a DUT. A similar PC probe employing a finer conical tip is being incorporated into a scanning force microscope in order to combine submicron spatial resolution with picosecond measurements of electrical signals.

This research was sponsored by the National Science Foundation through the Center for Ultrafast Optical Science under STC PHY 8920108 and by Fujitsu Laboratories, Ltd. Support was also provided by the Air Force Office of Scientific Research, Air Force Materiel Command, USAF, under Grant No. DOD-G-F49620-95-1-0227.

${ }^{1}$ J. A. Valdmanis and G. Mourou, IEEE J. Quantum Electron. 22, 69 (1986).

${ }^{2}$ J. Kim, S. Williamson, J. Nees, S. Wakana, and J. Whitaker, Appl. Phys. Lett. 62, 2268 (1993).

${ }^{3}$ T. Pfeifer, H.-M. Heiliger, E. Stein von Kamienski, H. G. Roskos, and H. Kurz, J. Opt. Soc. Am. B 11, 2547 (1994).

${ }^{4}$ M. Y. Frankel, J. F. Whitaker, and G. A. Mourou, IEEE J. Quantum Electron. 28, 2313 (1992).

${ }^{5}$ J. M. Chwalek and D. R. Dykaar, Rev. Sci. Instrum. 61, 1273 (1990).

${ }^{6}$ S. Gupta, J. F. Whitaker, and G. Mourou, IEEE J. Quantum Electron. 28, 2464 (1992)

${ }^{7}$ J. R. Hwang, H. J. Cheng, J. F. Whitaker, and J. V. Rudd, Appl. Phys. Lett. 68, 1464 (1996).

${ }^{8}$ J. R. Hwang, H. J. Cheng, J. F. Whitaker, and J. V. Rudd, Opt. Quantum Electron. 28, 961 (1996).

${ }^{9}$ R. K. Lai, J.-R. Hwang, J. Nees, T. B. Norris, and J. F. Whitaker, Appl. Phys. Lett. 69, 1843 (1996).

${ }^{10}$ D. H. Auston, IEEE J. Quantum Electron. 19, 639 (1983).

${ }^{11}$ D. H. Auston, in Ultrashort Laser Pulses: Generation and Applications, 2nd ed., edited by W. Kaiser (Springer, New York, 1993), p. 203. 